\title{
Generalization of Michell's solution to the plane problem theory of elasticity in polar coordinates in the event of a radially inhomogeneous body
}

\author{
V. I. Andreev \& N. Y. Cybin \\ Department of Strength of Materials, \\ Moscow State University of Civil Engineering, Russia
}

\begin{abstract}
In the plane problem of elasticity of inhomogeneous bodies problems are often found with the radial inhomogeneity occurring in the presence of axially symmetric physical fields (temperature, radiation, etc.). At the same time the plane problem itself can be two-dimensional. Of homogeneous bodies Michell's solution for Airy's stress function is the most well known. This solution is presented in the form of an infinite series in the trigonometric functions with constant coefficients. This article considers the statement of the problem in displacements, when the main unknown chosen functions are $u(r, \theta)$ and $v(r, \theta)$. The solution in displacements has the advantage that if the boundary conditions are in displacements it is not necessary to integrate Cauchy relations. Displacements are also represented in the form of series, but unlike in Michell's solutions the coefficients of trigonometric functions are also functions that depend on the radius. They are also solved in the example.
\end{abstract}

Keywords: Michell's solution, theory of elasticity, plane problem, inhomogeneity.

\section{Introduction}

This paper considers the method of solving the plane problem of the theory of elasticity of inhomogeneous bodies in polar coordinates. For the case of radial inhomogeneity there is a method of separation of variables which is a generalization of Michell's solutions for a homogeneous body [1]. 


\section{Main equations}

For the case where the mechanical characteristics of the material depend only on the radius the equations of the plane problem in polar coordinates are

$$
\begin{gathered}
(\lambda+2 \mu)\left(\frac{\partial^{2} u}{\partial r^{2}}+\frac{1}{r} \frac{\partial u}{\partial r}-\frac{u}{r^{2}}\right)+\frac{\mu}{r^{2}} \frac{\partial^{2} u}{\partial \theta^{2}}+\frac{\lambda+\mu}{r} \frac{\partial^{2} v}{\partial r \partial \theta}- \\
-\frac{\lambda+3 \mu}{r^{2}} \frac{\partial v}{\partial \theta}+\frac{\partial(\lambda+2 \mu)}{\partial r} \frac{\partial u}{\partial r}+\frac{1}{r} \frac{\partial \lambda}{\partial r}\left(u+\frac{\partial v}{\partial \theta}\right)-3 \frac{\partial}{\partial r}\left(K \varepsilon_{f}\right)+R=0 \\
\mu\left(\frac{\partial^{2} v}{\partial r^{2}}+\frac{1}{r} \frac{\partial v}{\partial r}-\frac{v}{r^{2}}\right)+\frac{\lambda+\mu}{r} \frac{\partial^{2} u}{\partial r \partial \theta}+\frac{\lambda+3 \mu}{r^{2}} \frac{\partial u}{\partial \theta}+ \\
+\frac{\lambda+2 \mu}{r^{2}} \frac{\partial^{2} v}{\partial \theta^{2}}+\frac{1}{r} \frac{\partial \mu}{\partial r}\left(\frac{\partial u}{\partial \theta}+r \frac{\partial v}{\partial r}-v\right)-\frac{3}{r} \frac{\partial}{\partial \theta}\left(K \varepsilon_{f}\right)+\Theta=0
\end{gathered}
$$

where $\lambda=\lambda(r), \mu=\mu(r), \varepsilon_{f}$ denotes forced (e.g. temperature) strains, $R, \Theta$ are mass forces. In the plane stress problem $\lambda$ and $\mu$ must be replaced by

$$
\lambda^{*}=\frac{2 \lambda \mu}{\lambda+2 \mu}=\frac{E v}{1-v^{2}} ; \quad \mu^{*}=\frac{2 K \mu}{\lambda+2 \mu}=\frac{E}{3(1-v)} .
$$

\section{Solution for homogeneous material}

If the mechanical properties of the material are constant it is known as Michell's solution for the Airy stress function [1]. In the notation of the coefficients according to [1], we can write the complete solution in the form:

$$
\begin{aligned}
& \Phi=a_{0} \ln r+b_{0} r^{2}+c_{0} r^{2} \ln r+d_{0} r^{2} \theta+a_{0}^{\prime} \theta+\frac{a_{1}}{2} r \theta \sin \theta+\left(b_{1} r^{3}+\right. \\
& \left.+a_{1}^{\prime} r^{-1}++b_{1}^{\prime} r \ln r\right) \cos \theta-\frac{c_{1}}{2} r \theta \cos \theta+\left(d_{1} r^{3}+c_{1}^{\prime} r^{-1}+\right. \\
& \left.+d_{1}^{\prime} r \ln r\right) \sin \theta+\sum_{n=2}^{\infty}\left(a_{n} r^{n}+b_{n} r^{n+2}+a_{n}^{\prime} r^{-n}+b_{n}^{\prime} r^{-n+2}\right) \cos n \theta+ \\
& +\sum_{n=2}^{\infty}\left(c_{n} r^{n}+d_{n} r^{n+2}+c_{n}^{\prime} r^{-n}+d_{n}^{\prime} r^{-n+2}\right) \sin n \theta .
\end{aligned}
$$

Stresses can be determined by known formulas:

$$
\sigma_{r}=\frac{1}{r} \frac{\partial \Phi}{\partial r}+\frac{1}{r^{2}} \frac{\partial^{2} \Phi}{\partial \theta^{2}} ; \quad \sigma_{\theta}=\frac{\partial^{2} \Phi}{\partial r^{2}} ; \quad \tau_{r \theta}=\frac{1}{r^{2}} \frac{\partial \Phi}{\partial \theta}-\frac{1}{r} \frac{\partial^{2} \Phi}{\partial r \partial \theta} .
$$

Having determined the deformation from Hooke's law and integrating Cauchy relations, we obtain expressions for displacements. For the case of plane stress state they are have the form [2]:

$$
E u=-a_{0} \frac{1+v}{r}+2 b_{0}(1-v) r+c_{0}[2(1-v) r \ln r-(1+v) r]+d_{0}(1-v) r \theta+
$$




$$
\begin{gathered}
+\left[\frac{a_{1}(1-v)}{2}+2 b^{\prime}\right] \theta \sin \theta-\left[\frac{c_{1}(1-v)}{2}+2 d_{1}^{\prime}\right] \theta \cos \theta+\left[c_{1} \ln r+d_{1}(1-3 v) r^{2}+\right. \\
\left.+c_{1}^{\prime}(1+v) r^{-2}+d_{1}^{\prime}(1-v) \ln r\right] \sin \theta+\left[a_{1} \ln r+b_{1}(1-3 v) r^{2}+a_{1}^{\prime}(1+v) r^{-2}+\right. \\
\left.+b_{1}^{\prime}(1-v) \ln r\right] \cos \theta+\sum_{n=2}^{\infty}\left\{a_{n} n(1+v) r^{n-1}+b_{n}[n-2+v(n+2)] r^{n+1}-\right. \\
\left.-a_{n}^{\prime} n(1+v) r^{-n-1}-b_{n}^{\prime}[n+2+v(n-2)] r^{-n+1}\right\} \cos n \theta-\sum_{n=2}^{\infty}\left\{{ }_{n} n(1+v) r^{n-1}+\right. \\
\left.+d_{n}[n-2+v(n+2)] r^{n+1}-c_{n}^{\prime} n(1+v) r^{-n-1}-d_{n}^{\prime}[n+2+v(n-2)] r^{-n+1}\right\} \sin n \theta \\
E v=4 c_{0} r \theta-4 d_{0} r \ln r-a_{0}^{\prime} \frac{1+v}{r}+\left[c_{1}(1-v) / 2+2 d_{1}^{\prime}\right] \theta \sin \theta+ \\
+\left[a_{1}(1-v) / 2+2 b_{1}^{\prime}\right] \theta \cos \theta-\left[a_{1}(2 \ln r+1+v) / 2-b_{1}(5+v) r^{2}-\right. \\
\left.-a_{1}^{\prime}(1+v) r^{-2}+b_{1}^{\prime}(1+v+\ln r-v \ln r)\right] \sin \theta+\left[c_{1}(2 \ln r+1+v) / 2-\right. \\
\left.\quad-d_{1}(5+v) r^{2}-c_{1}^{\prime}(1+v) r^{-2}+d_{1}^{\prime}(1+v+\ln r-v \ln r)\right] \cos \theta+ \\
+\sum_{n=2}^{\infty}\left\{a_{n} n(1+v) r^{n-1}+b_{n}[4+n(1-v)] r^{n+1}+a_{n}^{\prime} n(1+v) r^{-n-1}-\right. \\
\left.-b_{n}^{\prime}[4-n(1+v)] r^{-n+1}\right\} \sin n \theta-\sum_{n=2}^{\infty}\left\{c_{n} n(1+v) r^{n-1}+d_{n}[4+n(1-v)] r^{n+1}+\right. \\
\left.+c_{n}^{\prime} n(1+v) r^{-n-1}-d_{n}^{\prime}[4-n(1+v)] r^{-n+1}\right\} \cos n \theta
\end{gathered}
$$

In general, the solutions of equations (1) and (2) depend on the functions $\lambda(r, \theta), \mu(r, \theta), K(r, \theta)$ and cannot be written in closed form. In the following for the case when the mechanical properties together with their first derivatives are continuous functions of the radius, by the method of separation of variables equations (1) and (2) are reduced to systems of ordinary differential equations, which, by analogy with the Michell's generalized solution, are called generalized equations of the plane problem in polar coordinates.

\section{Generalized equations of the plane problem for a radially inhomogeneous body}

Using the analogy with the solutions (4) and (5) for a homogeneous material, we seek the solution of equations (1) and (2) in the form

$$
\begin{aligned}
& \left(\begin{array}{l}
u \\
v
\end{array}\right)=\left(\begin{array}{l}
\varphi_{0} \\
\psi_{0}
\end{array}\right)+\left(\begin{array}{l}
\varphi_{1} \\
\psi_{1}
\end{array}\right) \cdot \theta+\left(\begin{array}{l}
\varphi_{2} \\
\psi_{2}
\end{array}\right) \cdot \theta \sin \theta+\left(\begin{array}{l}
\varphi_{3} \\
\psi_{3}
\end{array}\right) \cdot \theta \cos \theta+ \\
& +\left(\begin{array}{l}
\varphi_{4} \\
\psi_{4}
\end{array}\right) \cdot \sin \theta+\left(\begin{array}{l}
\varphi_{5} \\
\psi_{5}
\end{array}\right) \cdot \cos \theta+\sum_{n=2}^{\infty}\left[\left(\begin{array}{c}
\varphi_{s n} \\
\psi_{s n}
\end{array}\right) \sin n \theta+\left(\begin{array}{c}
\varphi_{c n} \\
\psi_{c n}
\end{array}\right) \cos n \theta\right],
\end{aligned}
$$

where $\varphi_{0}, \ldots \psi_{c n}$ are the functions depending only on the radius. It is assumed that $\varepsilon_{f}(r, \theta), R(r, \theta), \Theta(r, \theta)$ also might also be presented by series similar to (6). 
Substituting (6) into (1) and (2) by equating to zero the sum of terms that do not contain $\theta$, as well as the amounts which are multiplied by $\theta, \theta \sin \theta$, and so on, leads to an infinite system of ordinary differential equations for the functions $\varphi_{i}, \psi_{i}$. Below are the equations for some functions from the expressions (6)

$$
\begin{aligned}
& (\lambda+2 \mu)\left(\varphi_{0}^{\prime \prime}+\frac{\varphi_{0}^{\prime}}{r}-\frac{\varphi_{0}}{r^{2}}\right)+\frac{\lambda+\mu}{r} \psi_{1}^{\prime}-\frac{\lambda+3 \mu}{r^{2}} \psi_{1}+ \\
& +\left(\lambda^{\prime}+2 \mu^{\prime}\right) \varphi_{0}^{\prime}+\frac{\lambda^{\prime}}{r}\left(\varphi_{0}+\psi_{1}\right)-3\left(K \varepsilon_{f, 0}\right)^{\prime}+R_{0}=0 ; \\
& \frac{\lambda+\mu}{r} \varphi_{1}^{\prime}+\frac{\lambda+3 \mu}{r^{2}} \varphi_{1}+\mu\left(\psi_{0}^{\prime \prime}+\frac{\psi_{0}^{\prime}}{r}-\frac{\psi_{0}}{r^{2}}\right)+ \\
& +\frac{\mu^{\prime}}{r}\left(\varphi_{1}+r \psi_{0}^{\prime}-\psi_{0}\right)-\frac{3 K}{r} \varepsilon_{f, 1}+T_{0}=0 ; \\
& (\lambda+2 \mu)\left(\varphi_{1}^{\prime \prime}+\frac{\varphi_{1}^{\prime}}{r}-\frac{\varphi_{1}}{r^{2}}\right)+\left(\lambda^{\prime}+2 \mu^{\prime}\right) \varphi_{1}^{\prime}++\frac{\lambda^{\prime}}{r} \varphi_{1}-3\left(K \varepsilon_{f, 1}\right)^{\prime}+R_{1}=0 ; \\
& \mu\left(\psi_{1}^{\prime \prime}+\frac{\psi_{1}^{\prime}}{r}-\frac{\psi_{1}}{r^{2}}\right)+\frac{\mu^{\prime}}{r}\left(r \psi_{1}^{\prime}-\psi_{1}\right)+T_{1}=0 ; \\
& (\lambda+2 \mu)\left(\varphi_{s n}^{\prime \prime}+\frac{\varphi_{s n}^{\prime}}{r}-\frac{\varphi_{s n}}{r^{2}}\right)-\frac{\mu n^{2}}{r^{2}} \varphi_{s n}-\frac{\lambda+\mu}{r} n \psi_{c n}^{\prime}+\frac{\lambda+3 \mu}{r^{2}} n \psi_{c n}+ \\
& +\left(\lambda^{\prime}+2 \mu^{\prime}\right) \varphi_{s n}^{\prime}+\frac{\lambda^{\prime}}{r}\left(\varphi_{s n}-n \psi_{c n}\right)-3\left(K \varepsilon_{f, s n}\right)^{\prime}+R_{s n}=0 ; \\
& -\frac{\lambda+\mu}{r} n \varphi_{c n}^{\prime}-\frac{\lambda+3 \mu}{r^{2}} n \varphi_{c n}+\mu\left(\psi_{s n}^{\prime \prime}+\frac{\psi_{s n}^{\prime}}{r}-\frac{\psi_{s n}}{r^{2}}\right)-\frac{\lambda+2 \mu}{r^{2}} n^{2} \psi_{s n}- \\
& -\frac{\mu^{\prime}}{r}\left(n \varphi_{c n}-r \psi_{s n}^{\prime}+\psi_{s n}\right)+\frac{3 K}{r} n \varepsilon_{f, c n}+T_{s n}=0 \text {; } \\
& (\lambda+2 \mu)\left(\varphi_{c n}^{\prime \prime}+\frac{\varphi_{c n}^{\prime}}{r}-\frac{\varphi_{c n}}{r^{2}}\right)-\frac{\mu n^{2}}{r^{2}} \varphi_{c n}+\frac{\lambda+\mu}{r} n \psi_{s n}^{\prime}-\frac{\lambda+3 \mu}{r^{2}} n \psi_{s n}+ \\
& +\left(\lambda^{\prime}+2 \mu^{\prime}\right) \varphi_{c n}^{\prime}+\frac{\lambda^{\prime}}{r}\left(\varphi_{c n}+n \psi_{s n}\right)-3\left(K \varepsilon_{f, c n}\right)^{\prime}+R_{c n}=0 ; \\
& \frac{\lambda+\mu}{r} n \varphi_{s n}^{\prime}+\frac{\lambda+3 \mu}{r^{2}} n \varphi_{s n}+\mu\left(\psi_{c n}^{\prime \prime}+\frac{\psi_{c n}^{\prime}}{r}-\frac{\psi_{c n}}{r^{2}}\right)-\frac{\lambda+2 \mu}{r^{2}} n^{2} \psi_{c n}+ \\
& +\frac{\mu^{\prime}}{r}\left(n \varphi_{s n}+r \psi_{c n}^{\prime}-\psi_{c n}\right)-\frac{3 K}{r} n \varepsilon_{f, s n}+T_{c n}=0 .
\end{aligned}
$$

In the above equations, the prime denotes differentiation with respect to the radius. Obtained system of equations is partially decomposed. For example, equations (11) and (14) form a closed system with respect to a pair of functions $\varphi_{s n}$ and $\psi_{c n}$, and equations (12) and (13) respectively to functions $\varphi_{c n}$ and 
$\psi_{s n}$. The rest of the functions in (6) can be found consistently from the corresponding equations. For example, from (9) and (10) we can find $\varphi_{1}$ and $\psi_{1}$ after which from (7) and (8) we can find $\varphi_{0}$ and $\psi_{0}$.

To equations (7)-(14) shall be added boundary conditions of any type. Formulas for stresses can be obtained from Hooke's law with Cauchy relations and expressions (6).

\section{Two analytical solutions}

\subsection{Pure torsion of thin ring}

Consider a thin ring fixed on the inner contour $(r=a)$, and on the outer $(r=b)$ loaded with uniform tangential forces with intensity $q$ (Fig. 1). Because the displacements $v$ do not dependent on $\theta$, the solution is sought in the form $v=\psi_{0}$. Other functions $\psi_{i}$ and all functions $\varphi_{i}$ are equal to zero. According to (8) $\psi_{0}$ satisfies the equation

$$
\psi_{0}^{\prime \prime}+\psi_{0}^{\prime}\left(\frac{1}{r}+\frac{\mu^{\prime}}{\mu}\right)-\frac{\psi_{0}}{r}\left(\frac{1}{r}+\frac{\mu^{\prime}}{\mu}\right)=0,
$$

and also to boundary conditions:

$$
r=a, \quad \psi_{0}=0 ; \quad r=b, \quad \tau=q \Rightarrow \mu\left(\psi_{0}^{\prime}-\frac{\psi_{0}}{r}\right)=q .
$$

It is easy to show that if $\mu=\mu_{0}=$ const is a solution of (15) then $\psi_{0}=C_{1} r+\frac{C_{2}}{r}$ which corresponds to the members of the formula (5), and comprising constants $c$ and $a_{0}^{\prime}$. By satisfying the conditions (16) in the case of a homogeneous material we obtain

$$
v^{(h o m)}=\frac{q b^{2}}{2 \mu_{0} a}\left(\frac{r}{a}-\frac{a}{r}\right) ; \quad \tau_{r \theta}^{(\text {hom })}=q\left(\frac{b}{r}\right)^{2} .
$$

Proceeding to the solution for the inhomogeneous material we will consider one of the simplest dependencies $\mu=\mu_{0}(r / a)^{\delta}$. In that event equation (15) is the Euler equation, the solution of which is

$$
\psi_{0}=C_{1} r+C_{2} r^{-\delta-1} .
$$

Having determined the constants from (16) we find $v$ and $\tau_{r \theta}$ :

$$
v^{(i n h)}=\frac{q b^{2}}{(\delta+2) \mu_{0} a}\left(\frac{r}{a}-\frac{a^{\delta+1}}{r^{\delta+1}}\right) ; \quad \tau_{r \theta}^{(i n h)}=q\left(\frac{b}{r}\right)^{2} .
$$




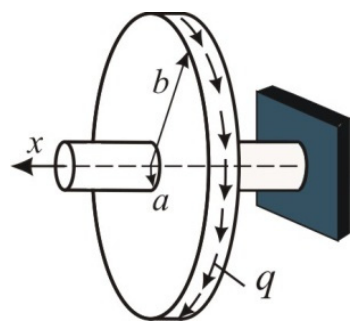

Figure 1: Design scheme.

In expressions (17) and (18) the superscripts refer respectively to the homogeneous and inhomogeneous material. Comparing the two solutions, we can see that the stresses in the ring do not depend on the inhomogeneity, which can be explained as follows. This problem is statically determinate. If you cut the ring the inner radius of which is $r_{0}>a$ (Fig. 2), the stresses on the inner contour of the ring can be calculated from the condition that the torque about the axis $x$ is equal to zero:

$$
\sum M(x)=q \cdot \Delta \cdot 2 \pi b \cdot b-\tau\left(r_{0}\right) \cdot \Delta \cdot 2 \pi r_{0} \cdot r_{0}=0 \Rightarrow \tau\left(r_{0}\right)=q b^{2} / r_{0}^{2} .
$$

Displacements in the two cases are different. The ratio of displacement of the outer contour points in the inhomogeneous and homogeneous rings is

$$
\frac{v^{(i n h)}(b)}{v^{(h o m)}(b)}=\frac{2\left(k^{\delta+2}-1\right)}{(\delta+2)\left(k^{2}-1\right) k^{\delta}},
$$

where $k=b / a$. Analysis shows that when $\delta>0$, which corresponds to toughening the material with increasing radius, this ratio is always less than unity.

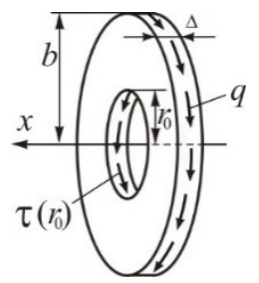

Figure 2: Equilibrium of the ring: $\Sigma M(x)=0$.

\subsection{Ring under the action of normal and shear loads}

Let us consider the problem of equilibrium in the thin ring when on the outer surface the loads are distributed:

$$
p=p_{0}(1+\cos 2 \theta) / 2 ; \quad q=p_{0} \sin 2 \theta / 2,
$$


and on the inner surface there are no loads (Fig. 3). In the case $a<<b$ it is the Kirsch's problem about stretching of the thin plate with a small circular hole.

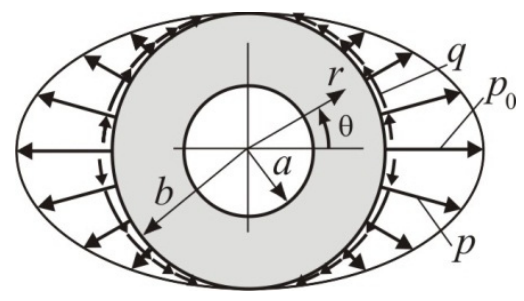

Figure 3: Calculation scheme.

Let the modulus of elasticity of the ring's material vary according to the power law

$$
E(r)=E_{0}\left(\frac{r}{a}\right)^{\delta}
$$

and Poisson ratio $\mathrm{v}=$ const.

To satisfy the boundary conditions

$$
\begin{array}{ll}
r=a, & \sigma_{r}=\tau_{r \theta}=0 ; \\
r=b, & \sigma_{r}=p(\theta), \quad \tau_{r \theta}=-q(\theta)
\end{array}
$$

in the expressions for the stresses we can restrict the terms containing the functions $\varphi_{0}, \psi_{1}, \varphi_{c 2}$ and $\psi_{s 2}$ :

$$
\begin{gathered}
\sigma_{r}=\frac{E}{(1+v)(1-v)}\left[\left(\varphi_{0}^{\prime}+v \frac{\varphi_{0}+\psi_{1}}{r}\right)+\left(\varphi_{c 2}^{\prime}+v \frac{\varphi_{c 2}+2 \psi_{s 2}}{r}\right) \cdot \cos 2 \theta\right] \\
\sigma_{\theta}=\frac{E}{(1+v)(1-v)}\left[\left(\frac{\varphi_{0}+\psi_{1}}{r}+v \varphi_{0}^{\prime}\right)+\left(\frac{\varphi_{c 2}+2 \psi_{s 2}}{r}+v \varphi_{c 2}^{\prime}\right) \cdot \cos 2 \theta\right] \\
\tau_{r \theta}=\frac{E}{2(1+v)}\left[\left(\psi_{1}^{\prime}-\frac{\psi_{1}}{r}\right) \cdot \theta+\left(\psi_{s 2}^{\prime}-\frac{\psi_{s 2}+2 \varphi_{c 2}}{r}\right) \cdot \sin 2 \theta\right]
\end{gathered}
$$

For determining functions $\varphi_{0}, \psi_{1}, \varphi_{c 2}$ and $\psi_{s 2}$ it is necessary to consider equations (7), (10), (12) and (13) for $n=2$, taking into account $\varepsilon_{\mathrm{B}}=R=\Theta=0$. To go from a plane strain to plane stress state we will replace $\lambda$ with $\lambda^{*}=E v /\left(1-v^{2}\right)$.

From the condition of uniqueness, the solution must not contain $\theta$. Thus, from the third equality (22) it follows that

$$
\psi_{1}^{\prime}-\frac{\psi_{1}}{r}=0
$$


By using this equality equation (10) takes the form

$$
\psi_{1}^{\prime \prime}=0 \text {. }
$$

The integral of this equation is a linear function which comes is constant at $r=a, b$. Taking into account that boundary condition for $\tau_{r \theta}$, equation (19) does not contain a constant so we must assume $\psi_{1}=0$. Then equation (7) is simplified:

$$
\left(\lambda^{*}+2 \mu\right)\left(\varphi_{0}^{\prime \prime}+\frac{\varphi_{0}^{\prime}}{r}-\frac{\varphi_{0}}{r^{2}}\right)+\left[\left(\lambda^{*}\right)^{\prime}+2 \mu^{\prime}\right] \varphi_{0}^{\prime}+\frac{\left(\lambda^{*}\right)^{\prime}}{r} \varphi_{0}=0 .
$$

Thus, the problem can be separated into two problems: determination using equation (23) for the axisymmetric part of the solution and the determination from equations (12) and (13) which depends on $\theta$. Substitution of (20) into these equations leads them to the form

$$
\begin{gathered}
\varphi_{0}^{\prime \prime}+(1+\delta) \frac{\varphi_{0}^{\prime}}{r}+(\delta v-1) \frac{\varphi_{0}}{r^{2}}=0 \\
\psi_{s 2}^{\prime \prime}+(1+\delta) \frac{\psi_{s 2}^{\prime}}{r}-\left(\frac{9-v}{1-v}+\delta\right) \frac{\psi_{s 2}}{r^{2}}-\frac{2(1+v)}{1-v} \frac{\varphi_{c 2}^{\prime}}{r}-\left(\frac{6-2 v}{1-v}+2 \delta\right) \frac{\varphi_{c 2}}{r^{2}}=0 \\
\varphi_{c 2}^{\prime \prime}+(1+\delta) \frac{\varphi_{c 2}^{\prime}}{r}+(\delta v-3+2 v) \frac{\varphi_{c 2}}{r^{2}}+(1+v) \frac{\psi_{s 2}^{\prime}}{r}-(3-v-2 \delta v) \frac{\psi_{s 2}}{r^{2}}=0 .
\end{gathered}
$$

Solution of equation (24) is the function [3]:

$$
\varphi_{0}=C_{1} r^{\frac{1-\alpha+\beta}{2}}+C_{2} r^{\frac{1-\alpha-\beta}{2}},
$$

where $\alpha=(1+\delta), \beta=\sqrt{(1-\alpha)^{2}-4(\delta v-1)}$.

Integration constants appearing in (27) can be found from the boundary conditions for the axisymmetric component of the external load:

$$
r=a, \quad \sigma_{r}=0 ; \quad r=b, \quad \sigma_{r}=p_{0} / 2 .
$$

The system of two ordinary differential equations (25) and (26) can be reduced to a single fourth-order equation:

$$
\begin{aligned}
& r^{4} \varphi_{c 2}^{I V}+(6+2 \delta) r^{3} \varphi_{c 2}^{\prime \prime \prime}+\left(5 \delta+\delta^{2}+v \delta-3\right) r^{2} \varphi_{c 2}^{\prime \prime}+ \\
& +\left(v \delta^{2}+v \delta-9 \delta-9\right) r \varphi_{c 2}^{\prime}+\left(3 v \delta^{2}+3 v \delta-3 \delta+9\right) \varphi_{c 2}=0 .
\end{aligned}
$$

The obtained equation can be reduced to a differential equation with constant coefficients by introducing variable $t$ using dependence $r=\exp (t)$ : 


$$
\begin{aligned}
& \frac{d^{4} \varphi_{c 2}}{d t^{4}}+2 \delta \frac{d^{3} \varphi_{c 2}}{d t^{3}}+\left(\delta^{2}+v \delta-\delta-10\right) \frac{d^{2} \varphi_{c 2}}{d t^{2}}+ \\
& +\left(v \delta^{2}-\delta^{2}-10 \delta\right) \frac{d \varphi_{c 2}}{d t}+\left(3 v \delta^{2}+3 v \delta-3 \delta+9\right) \varphi_{c 2}=0
\end{aligned}
$$

The characteristic equation corresponding to the obtained equation is

$$
\begin{aligned}
l^{4}+ & 2 \delta l^{3}+\left(\delta^{2}+v \delta-10\right) l^{2}+\left(v \delta^{2}-\delta^{2}-10 \delta\right) l+ \\
& +3 v \delta^{2}+3 v \delta-3 \delta+9=0
\end{aligned}
$$

Using substitution $\eta=l^{2}+\delta l$ this equation can be reduced to a quadratic equation:

$$
\eta^{2}+(v \delta-\delta-10) \eta+3 v \delta^{2}+3 v \delta-3 \delta+9=0
$$

The final solution of equation (29) is represented as

$$
\varphi_{c 2}=\sum_{i=1}^{4} D_{i} e^{l t}
$$

Constants $D_{i}$ determined from the boundary conditions for the nonaxisymmetric component:

$$
\begin{gathered}
r=a, \quad \sigma_{r}=\tau_{r \theta}=0 \\
r=b, \quad \sigma_{r}=p_{0} \cos 2 \theta / 2, \\
\tau_{r \theta}=-p_{0} \sin 2 \theta / 2 .
\end{gathered}
$$

Function $\psi_{s 2}$ can be found from eqn (25). Below is an example of the calculation performed for the following initial data: $\delta=-1 ; b / a=2 ; v=1 / 3$; $E=2 \cdot 10^{4} \mathrm{MPa}$.

Fig. 4 shows diagrams of stresses $\sigma_{\theta}$ along three radial directions. You may see that greatest differences of stress for inhomogeneous and homogeneous materials do not exceed $20 \%$. Fig. 5 shows diagrams of the displacements of the inner contour points of the ring along the angular coordinate. It can be noted that displacement in the inhomogeneous ring is greater than in the homogeneous ring. This is due to the fact that at $\delta=-1$ the modulus decreases from the inner to the outer contour twice, and this leads to a decrease in the total ring stiffness. 


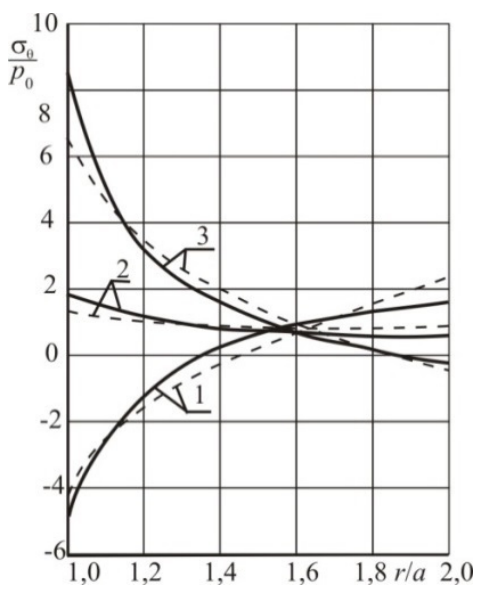

Figure 4: Stresses $\sigma_{\theta}$ in the ring. $1-\theta=0 ; 2-\theta=\pi / 4 ; 3-\theta=\pi / 2$; inhomogeneous material, - - - homogeneous material.

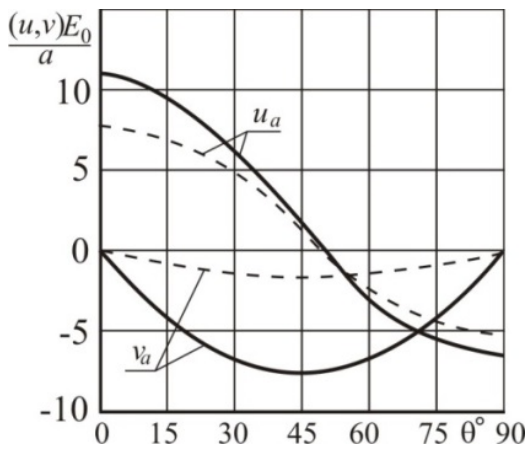

Figure 5: Displacements of inner contour of the ring. inhomogeneous material, - - - homogeneous material.

\section{Numerical-analytical method of solution}

If the dependencies $E(r)$ and $v(r)$ are complex enough then it is not possible to obtain an analytical solution. In this case it is necessary to use numerical integration methods. Reduction of partial differential equations to a system of ordinary differential equations and their subsequent numerical solution is called the numerical-analytical method (this method is sometimes called the semianalytic method). 
The above equations (7)-(14) and the equations for the remaining functions $\varphi_{i}$ and $\psi_{i}$ included in expressions (6) can be divided into groups of two second-order equations for a pair of functions. Two second-order equations may be reduced to the four equations of the first order, and the resulting system of four equations can be solved numerically using one of the computer systems such as Matlab. In the following we give an example of an appropriate calculation.

Here is the solution of the problem discussed in Section 5.2. We reduce equations (12) and (13) to a system of four first-order equations. We introduce the notation

$$
y_{1}=\varphi_{c 2}, y_{2}=\varphi_{c 2}^{\prime}, y_{3}=\psi_{s 2}, y_{4}=\psi_{s 2}^{\prime}
$$

Then equations (12) and (13) can be written as

$$
\begin{aligned}
& -\frac{\lambda+\mu}{r} n y_{2}-\frac{\lambda+3 \mu}{r^{2}} n y_{1}+\mu\left(y_{4}^{\prime}+\frac{y_{4}}{r}-\frac{y_{3}}{r^{2}}\right)-\frac{\lambda+2 \mu}{r^{2}} n^{2} y_{3}- \\
& -\frac{\mu^{\prime}}{r}\left(n y_{1}-r y_{4}+y_{3}\right)+\frac{3 K}{r} n \varepsilon_{\mathrm{f}, c n}+T_{s n}=0 ; \\
& (\lambda+2 \mu)\left(y_{2}^{\prime}+\frac{y_{2}}{r}-\frac{y_{1}}{r^{2}}\right)-\frac{\mu n^{2}}{r^{2}} y_{1}+\frac{\lambda+\mu}{r} n y_{4}-\frac{\lambda+3 \mu}{r^{2}} n y_{3}+ \\
& +\left(\lambda^{\prime}+2 \mu^{\prime}\right) y_{2}+\frac{\lambda^{\prime}}{r}\left(y_{1}+n y_{3}\right)-3\left(K \varepsilon_{\mathrm{f}, c n}\right)^{\prime}+R_{c n}=0 ;
\end{aligned}
$$

Adding to these two equations the equalities

$$
y_{1}^{\prime}=y_{2} ; \quad y_{3}^{\prime}=y_{4},
$$

which follow from (31), we receive the system of four first-order equations.

Boundary conditions for non-axisymmetric component (30) can be written as

$$
\begin{aligned}
& r=a, \quad y_{2}+v \frac{y_{1}+2 y_{3}}{a}=0 ; \quad y_{4}-\frac{y_{3}+2 y_{1}}{a}=0 . \\
& r=b, \quad\left\{\begin{array}{c}
\frac{E}{(1+v)(1-v)}\left(y_{2}+v \frac{y_{1}+2 y_{3}}{b}\right)=\frac{p_{0}}{2} ; \\
\frac{E}{2(1+v)}\left(y_{4}-\frac{y_{3}+2 y_{1}}{b}\right)=-\frac{p_{0}}{2} .
\end{array}\right.
\end{aligned}
$$

Thus equations (32)-(34) with boundary conditions (35) and (36) represent the boundary problem for the non-axisymmetric part of the solution.

Similarly, in view of $\psi_{1}=0$ the boundary value problem can be presented for the axisymmetric component:

$$
\varphi_{0}^{\prime \prime}+(1+\delta) \frac{\varphi_{0}^{\prime}}{r}+(\delta v-1) \frac{\varphi_{0}}{r^{2}}=0 ;
$$




$$
\begin{gathered}
r=a, \quad \varphi_{0}^{\prime}+v \frac{\varphi_{0}}{a}=0 ; \\
r=b, \quad \frac{E}{(1+v)(1-v)}\left(\varphi_{0}^{\prime}+v \frac{\varphi_{0}+\psi_{1}}{r}\right)=\frac{p_{0}}{2}
\end{gathered}
$$

Equation (37) with boundary conditions (38) is also easily solved using any software package.

To determine the accuracy of the numerical-analytic methods we performed calculations of the same sample as in Section 5.2 for the same initial data. Interval $[a, b]$ is divided into 100 steps. Table 1 shows the comparative values of the stresses in an inhomogeneous ring when $\theta=45^{\circ}$ obtained by analytical and numerical calculations.

Table 1: $\quad$ Stresses in the ring.

\begin{tabular}{|c|c|c|c|c|}
\hline \multirow{2}{*}{$r / a$} & \multicolumn{2}{|c|}{$\begin{array}{c}\text { Analytical } \\
\text { calculation }\end{array}$} & \multicolumn{2}{c|}{$\begin{array}{c}\text { Numerical } \\
\text { calculation }\end{array}$} \\
\cline { 2 - 5 } & $\sigma_{r}$ & $\tau_{r \theta}$ & $\sigma_{r}$ & $\tau_{r \theta}$ \\
\hline 1.0 & 0.0 & 0.0 & 0.0 & 0.0 \\
\hline 1.2 & 0.252 & -1.266 & 0.263 & -1.296 \\
\hline 1.4 & 0.377 & -1.338 & 0.378 & -1.363 \\
\hline 1.6 & 0.444 & -1.108 & 0.444 & -1.121 \\
\hline 1.8 & 0.480 & -0.807 & 0.480 & -0.813 \\
\hline 2.0 & 2.000 & -0.500 & 2.000 & -0.500 \\
\hline
\end{tabular}

Comparison of the results obtained by the two methods suggests a fairly high precision of the numerical-analytic method.

\section{Conclusions}

The developed method of separation of variables in the plane problem in aggregates with a numerical method for solving a system of ordinary differential equations allows solutions to be obtained for a wide range of problems for the radially inhomogeneous bodies. Such problems are encountered in heat and power generation, the construction of underground facilities and other areas of technology in the presence of temperature [4], radiation [5] and moisture [6-8]. The solution of such problems, taking into account heterogeneity, clarifies the stress-strain state of structures, which may lead to their optimization and to improvements in efficiency [9-11].

\section{Acknowledgement}

This work was supported by the Ministry of Education and Science of Russia under grant number № 7.2122.2014/K. 


\section{References}

[1] Timoshenko, S. \& Goodier J.N., Theory of Elasticity, McGraw-Hill Book Company: New York, Toronto and London, p. 116, 1951.

[2] Andreev V.I. About one method of solving of plane problem of the theory of elasticity for radial inhomogeneous body, Applied Mechanics: (Kiev), 23(4), pp. 16-23, 1987.

[3] Kamke E. Diferentialgleichungen, Leipzig, 1959.

[4] Andreev V.I., Minayeva A.S. Modeling of equal-stressed cylinder exposed power and thermal stresses, International Journal for Computational Civil and Structural Engineering. Volume 7, Issue 1, pp. 71-75, 2011.

[5] Andreev V.I., Dubrovskiy I.A. The irradiation effect on the stress state of a concrete shells, International Journal for Computational Civil and Structural Engineering Volume 6, Issue 1,2 ; pp. 39-44, 2010.

[6] Andreev V.I., Avershyev A.S. Stationary Problem of Moisture-elasticity for Inhomogeneous thick-walled Shells, Advanced Materials Research, Vols. 671-674, pp. 571-575, Trans Tech Publications, Switzerland, 2013.

[7] Andreev V.I., Avershyev A.S. Nonstationary problem moisture elasticity for nonhomogeneous hollow thick-walled cylinder, Transactions of International Conference on Fluid Structure Interaction, pp. 123-132 WIT Press, 2013.

[8] Andreev V.I., Avershyev A.S. About Influence of Moisture on Stress State of Soil taking into account Inhomogeneity, International Journal for Computational Civil and Structural Engineering №9, Iss. 3-4, pp. 14-20, 2013.

[9] Andreev V.I. Optimization of thick-walled shells based on solutions of inverse problems of the elastic theory for inhomogeneous bodies. Computer Aided Optimum Design in Engineering XII, WIT Press, pp. 189-201, 2012.

[10] Andreev V.I. About one way of optimization of the thick-walled shells, Applied Mechanics and Materials, Vols. 166-169, pp. 354-358, Trans Tech Publications, Switzerland, 2012.

[11] Andreev V.I. Inverse problems of the inhomogeneous theory of elasticity for thick-walled shells, Computational Methods and Experimental Measurements, Vol. 2, No 2, pp. 202-216, WIT Press, 2014. 